\title{
A survey: effects of different biofeedback system analysis with flutter device in respiratory disorders.
}

\author{
Rekha $K^{1^{*}}$, Anandh Vaiyapuri², Samuel Sundar Doss $\mathbf{D}^{3}$ \\ ${ }^{1}$ Saveetha College of Physiotherapy, Thandalam, Chennai, Tamil Nadu, India \\ ${ }^{2}$ Department of Physical Therapy and Health Rehabilitation, College of Applied Medical Sciences, Majmaah \\ University, Majmaah, Saudi Arabia \\ ${ }^{3}$ Department of Physiology, Saveetha Medical College, Thandalam, Chennai, Tamil Nadu, India
}

\begin{abstract}
Recently, patients with respiratory diseases utilize different devices, which help the ejection of mucus from the airways and the change of pulmonary function. The point of the present investigation is to find out the effectiveness of the present devices of respiratory physiotherapy, as it originates from the survey of writing. The current devices of physiotherapy for patients with respiratory diseases are introduced as an option therapy strategy or a supplemental therapy and they can persuade patients to apply therapy without anyone else. The biofeedback techniques utilized as a part of rehabilitation depend on biomechanical measurements and estimations of the physiological systems of the body. The physiological frameworks of the body which can be measured to give biofeedback are the neuromuscular system, the respiratory system, and the cardiovascular system. This paper inspected distinctive articles in totally 35 are taken from standard journals, for example, IEEE, Science direct, PubMed and Pedro about the biofeedback devices for the respiratory system, airway choice and different devices and the performance of the techniques are analysed. Likewise, the quantity of extensive scale considers inspecting the utilization of respiratory biofeedback in rehabilitation is restricted. Estimations of development, postural control, and force output can be made by utilizing various different devices and used to express biomechanical biofeedback.
\end{abstract}

Keywords: Biofeedback, Respiratory training, Hear rate, Devices, Techniques, Airway clearance, Physiotherapy.

Accepted on December 26, 2017

\section{Introduction}

The advance of escalated care medicine has significantly enhanced survival of basically sick patients, particularly in patients with Acute Respiratory Distress Syndrome (ARDS) [1]. This improved endurance is, however, in many cases related with common reconditioning, muscle deficiency, dyspnea, gloom, nervousness and diminished health-related personal satisfaction after Intensive Care Unit (ICU) discharge [2]. Reconditioning and particularly muscle weakness are recommended to have an imperative part in the disabled long drag valuable status in survivors of basic sickness [3]. Enhancing the health of individuals through pulmonary rehabilitation makes a system that is proactive and centered around keeping a man as healthy as could be allowed. The way of life of pulmonary rehabilitation acknowledges that a noteworthy debilitation is available which can't be enhanced by ordinary restorative treatment. This hindrance is that of demolding because of idleness. An artificial airway worked for mechanical ventilation advances amassing of emissions in patients' airway and decreases patients' capacity to hack out discharges in airways. Non-intrusive treatment contributes towards evaluating and treating different parts of the respiratory issue, for example, wind stream deterrent, bodily fluid maintenance, changes in ventilator pump work, dyspnea, weakened exercise execution, and personal satisfaction. The impacts of the flutter valve TM on respiratory mechanics to patients with bronchiectasis have been assessed by constrained spirometer $[4,5]$. This approach, nonetheless, does not permit the portrayal of bodily fluid transportation along the aviation routes [6]. Beneath area talked about the biofeedback system, airway clearance, et cetera.

\section{Biofeedback systems}

The expression "biofeedback" alludes to the instrumentation or training process that enables biologic data to be recorded, shown, and conveyed back to an individual, enabling the person to make alterations in physiologic procedures that may promote health or execution. The biofeedback circle begins at the sensor that sweeps the patient's breathing musicality. The sensor is associated with a speaker that changes over the 
electrical motivations into acoustical and visual outputs. These outputs are exchanged to the patient by a headpiece, which is comprised of an eye shield with lights and stereo headphones. Output increases and reduction in volume as well as a force as the patient breathes in and breathes out. Preparing regularly starts with a session in which a short mental stress test is trailed by a time of unwinding while physiologic parameters are recorded and shown [4]. Biofeedback training can include various physiologic modalities, including persons which reject autonomic sensory system excitement, for example, skin conductance and heart rate inconstancy, and persons that are not entirely connected with autonomic action, for example, surface muscle pressure. Amid a solitary session of biofeedback training, the mentor may chip away at a solitary parameter or switch between parameters, contingent upon how much advance is being made with each. By and large process to basic parameters to begin with, for example, respiratory rate, finger temperature, and skin conductance, proceeding onward to surface muscle strain, heart rate, and inevitably heart rate fluctuation, which is a more mind-boggling idea and all the more effortlessly saw later in the training procedure $[7,8]$.

\section{Airway clearance devices}

Airway Clearance Techniques (ACTs) [9], normally performed by respiratory physiotherapists, are proposed to help secretion mobilization and expectoration and to moderate difficulties related to discharge maintenance. High-recurrence Airway clearance systems fall into general classes: unassisted and assisted. The meaning of "assist" in this setting is that the respiratory device (e.g. ventilator) works on the respiratory system, as demonstrated by an expansion in Trans-respiratory weight, related to stream the aspiratory way, or a lessening in Trans-respiratory pressure, related to stream the expiratory way. Early therapy can improve recuperation and personal satisfaction [10] and activities to expel sputum from the airways (airway Clearance systems') might be essential. ACTs work using control of lung volumes, a gas stream, pneumonic weights and compressive strengths to shear sputum along the airways lumen towards the mouth [11]. This procedure is fundamental for viable clearance of emissions in conditions where mucociliary brokenness is available, for example, Chronic Obstructive Pneumonic Disorder (COPD). Along these lines, to decide if tiotropium influences bodily fluid hypersecretion and airway clearance, we examined patients with COPD experience issues in sputum expectoration and hack indications [12].

\section{Flutter in pulmonary disorders}

The flutter device was created in Switzerland and consolidates positive expiratory pressure therapy with high-frequency motions inside the airway. It is a controlled vibration system which produces positive expiratory pressure and cyclic swaying of the airways amid termination. The flutter device is a compact device intended to help clear mucus in patients with lung issue [13]. The guideline behind this device is that exhalation into the flutter valve causes a steel ball-bearing to sway at a high recurrence, bringing about the vibration of the airways and discontinuous positive expiratory pressure, to encourage mucus expectoration. Lapse ought to be moderate through the flutter valve, causing motions of the steel ball inside the cone of the flutter. Patients apply rehashed exhalations through the flutter valve [7].

\section{Literature Review Analysis}

Analysts have demonstrated that psychological factors, for example, stress can influence physical health, and can add to the advancement of different side effects of word-related sick health and of the physical and psychological issue, for example, sleep deprivation, tension, and mellow dejection. Literature survey investigation inspected the diverse journal papers in biofeedback system, airway clearance system, Flutter in pulmonary disorders and some different procedures.

\section{Respiratory biofeedback system}

Biofeedback is a compelling procedure through which patients train themselves to get an arrangement of abilities, the learning of which is expounded through the information given by a biofeedback device. As a health mediation procedure, biofeedback is notable to encourage treatment for a wide assortment of disarranges with a psychosomatic part, including asthma, cardiovascular diseases, hypertension, encephalopathies, and tension.

A few examinations have demonstrated that these breathing activities can constitute an effective treatment in numerous clinical settings like cardiovascular illnesses, asthma, fibromyalgia or post-awful anxiety. Such a non-meddling restorative arrangement should be performed on an 8 to $10 \mathrm{w}$ time frame by [1]. The author anticipated another rearranged smartphone based arrangement enabling individuals to handle effective heart intelligence biofeedback works out. In view of photoplethysmographic imaging through the Smartphone camera, this sensor-less innovation permits controlling cardiovascular intelligibility biofeedback practices through a streamlined heart rate changeability algorithm.

The inventor Liu et al. [2] presented a wearable device for biofeedback application in view of the Body Sensor Networks (BSN). The biofeedback device has produced integrates the heart rate checking belt with conductive texture and the biofeedback gadget with breath belt. A wearable respiratory biofeedback framework was for starters investigated in light of the BSN stage. The BSN equipment stage was intended to be scaled down and stackable.

Biofeedback device essentially utilized perceptions to show physiological information to the client by Bhandari et al. [14]. This examination has proposed a mediation that consolidates the advantages of biofeedback and music to enable clients to manage their anxiety reaction while playing out a visual errand. This approach empowered moderate breathing by altering the nature of the music in light of the client's breathing rate. Assess the mediation on a design with music and soundrelated biofeedback as free factors. Our outcomes demonstrate 
that our music-biofeedback mediation prompts bring down excitement (decreased electro-dermal movement and expanded heart rate changeability) than music alone, sound-related biofeedback alone and a control condition.

Chronic anxiety practiced in effective life produces an autonomic dysregulation, which in line brings about high resting heart rate and circulatory strain, and additionally lessened vagal tone by Munafo et al. [15]. Have projected a narrative approach that adventures focused biofeedback on diminishing harmful psychological and physiological results of stress in elite work environments. It's contrasted standard with aggressive biofeedback, demonstrating that conflict upgrades training viability in advancing health and re-establishing heart autonomic adjust.

As per Grishin et al.'s [16] work biofeedback is utilized as a part of the treatment of post-traumatic stretch issue and nervousness issue. Before Coronary Arterial Bypass Graft (CABG) surgery all patients $(n=93$, matured 40 to 77 y were isolated into three gatherings in the wake of performing capnography and trial course of Respiratory Biofeedback (RB). The main gathering (usual care or USU gathering, $n=48$ ) comprised of patients who declined extra mental help and RB, the second gathering comprised of patients who consented to get mental help (PSY gathering, $n=25$ ), and the third gathering were patients who acknowledged RB with mental help (RB gathering, $n=20)$. The errand of the $R B$ training for the members was to keep up the end-tidal partial pressure of carbon dioxide $10-20 \%$ over the underlying level. RB gives off an impression of being a plausible technique for recovery of the patients experiencing CABG surgery amid hospital stay.

Physical therapy is included in the non-therapeutic treatment of patients with intense and perpetual respiratory diseases by Gosselink [17], including obstructive and prohibitive pneumonic diseases, patients with neuromuscular disarranges, patients conceded for real surgery and patients with basic sickness in escalated mind. Physical therapy contributes towards evaluating and treating different parts of the respiratory issue, for example, wind current hindrance, bodily fluid maintenance, and changes in ventilatory pump work, dyspnea, disabled exercise execution and personal satisfaction. Evaluation of patients is basic to recognize quiet attributes that permit fitting determination of treatment modalities giving ideal energy and adequacy.

An advanced apparatus like biofeedback is less frequently attempted as an appraisal device to measure the feasibility of a deeply rooted system like alternate nostril breathing (NadiShodhana Pranayama) by Sudhir et al. [18]. The instrument had the compositing programming to portray general unwinding rate in light of these parameters in the conclusive report. The gathering was regulated to repeat alternate nostril breathing at 20 min every day for 3 months. The parameters were re-surveyed and looked at. Moreover, saw stress scale was managed to the investigation subjects both pre and post-mediation to note changes in the scores. There was critical unwinding accomplished by the subjects following pranayama for 3 months when contrasted with pattern $(p<0.005)$. Seen stress scores likewise diminished altogether after Pranayama $(\mathrm{p}<0.001)$. This inferred interchange nostril breathing was a viable system of diminishing stress and Biofeedback is valuable as an evaluation apparatus separated from being a treatment methodology in stress-related conditions.

The biofeedback techniques utilized as a part of recovery depend on biomechanical estimations and assessment of the physiological frameworks of the body by Oonagh et al. [19]. The physiological frameworks of the body which can be deliberated to give biofeedback are the neuromuscular framework, the respiratory framework, and the cardiovascular framework. Neuromuscular biofeedback techniques incorporate Electromyography (EMG) biofeedback and continuous ultrasound imaging (RTUS) biofeedback. Sorts of biomechanical biofeedback incorporate constraint plate frameworks, electro-goniometry, pressure biofeedback and camera-based frameworks, however, the confirmation for these is restricted. Biofeedback is usually conveyed by utilizing visual showcases, acoustic or haptic signs, however, more as of late Virtual Reality (VR) or exergaming innovation have been utilized as biofeedback signals. VR and exergaming innovation have been basically examined in post-CVA restoration, nonetheless, later work has demonstrated this kind of biofeedback to be powerful in enhancing exercise procedure in musculoskeletal populaces.

The present investigation by Marianna et al. [20] was intended for assessing the viability of a Respiratory Sinus Arrhythmia (RSA) Biofeedback (BF) intercession on mental and physiological results, in chiefs with abnormal state work obligations. Thirty-one administrators driving exceptional private or open organizations were arbitrarily appointed to either an RSA-BF training (RSA-BF; $\mathrm{N}=16$ ) or a control gathering $(\mathrm{N}=15)$. The RSA-BF training comprised of five weeks after week 45 min sessions, intended to build RSA, while controls needed to give an everyday push journal once per week. Subsequent to the training, administrators in the two gatherings revealed diminished heart rate very still, bring down nervousness levels and change in health associated personal satisfaction.

As the Heart Rate Variability (HRV) is accepted by a wide margin as the best pointer of autonomic sensory system action, this framework was utilized the spectral examination parameter of HRV, LF/HF proportion, as biofeedback methodology to show the autonomic sensory system state [21]. The framework is created on the Lab View stage and can be utilized to enable individuals to deliberately control the autonomic framework, to maintain the harmony amongst thoughtful and parasympathetic sensory system and subsequently reduce the stress.

\section{Airway clearance in respiratory system}

There is confirm that airway mucus hypersecretion is related to the seriousness and anticipation of COPD, however, the side effects are for the most part hard to treated by Etsuko [22]. In an open, non-controlled examination, this paper analysed the impact of the anticholinergic operator tiotropium on airway 
mucus hypersecretion in 22 COPD patients. Following a $4 \mathrm{w}$ running in period, the patients got $18 \mathrm{mg}$ of tiotropium once day by day conveyed through the handihaler for two months, while side effects and their effect related with sputum were scored by hack and sputum evaluation survey (CASA-Q). At w 0 and $\mathrm{w} 8$, spirometry was performed earlier and $30 \mathrm{~min}$ after the organization of albuterol. To test the impact of tiotropium on airway mucociliary transport, nasal clearance time was measured. Tiotropium diminishes side effects related to sputum in COPD patients, an impact that might be identified with the hindrance of airway clearance bodily fluid hypersecretion and change of airway mucociliary freedom.

To distinguish ACTs are used to treat patients with intense Escalation of Chronic Obstructive Pulmonary Disease (AECOPD) and recognize factors supporting their use, including advisors' learning of the writing. Reason designed a survey measuring the self-detailed rate of ACT remedy; view of ACT signs, points, significance and viability; factors affecting ACT decision; and learning of the proof. The principle factors impacting ACT decision were safety measures/contraindications to singular methods (148/189, $78 \%)$ and level of dyspnoea (136/189, 72\%). The essential point of ACT remedy was to clear sputum (178/189, 94\%). Comprehension of the proof for ACTs in AECOPDs was blended, with $43 \%$ referring to it as strong, 30\% uncertain and $19 \%$ vague.

Poor emission clearance by Chulee et al. [23] is a significant issue for intubated patients prompting lung intricacies and late weaning. To assess the adequacy and security of another gadget (Breathe MAX) that humidifies and wavers motivated air to build emission clearance in mechanically ventilated patients. Mediations comprised of unconstrained profound breathing with (treatment) and without (sham) humidification and wavering of forced air. Airway discharges were suctioned for $3 \mathrm{~h}$ previously, then after the fact every mediation and wet weight and consistency resoluted.

Airway clearance strategies incorporate positive expiratory pressure, normally utilized as a part of the clinical practice, and a lately presented impermanent positive expiratory pressure device called UNIKO by Francesco et al. It was misty which one gives the best advantage to patients. The point of this observational $4 \mathrm{y}$ research was to reflectively look at the adequacy of any particular signs for brief positive expiratory pressure contrasted with positive expiratory pressure in a standard recovery program gathered data from 162 subjects (107 guys, mean age $70 \pm 9 \mathrm{y}, 97$ with essential diagnosis of incessant obstructive pulmonary illness, 65 with bronchiectasis), 51 treated with impermanent positive expiratory pressure and 111 with positive expiratory pressure. Randomized clinical trials are important to affirm our preparatory outcomes demonstrating that distinctive subgroups/phenotypes can profit more from one kind of treatment.

Mechanically-ventilated patients in the emergency unit may experience the ill effects of held emissions from a few causes [24]. Airway clearance systems can possibly enhance mucociliary clearance by lessening mucus stopping and upgrading the expulsion of discharges, including inflammatory cells and microorganisms. This short audit portrayed late advance in airway clearance administration in ICU patients. Along these lines, randomized examinations, with strong clinical short and long-haul result measures, were required. Meanwhile, to pick a system, parental figures ought to consider the pathophysiologic method of reasoning for the restorative utilize, the gear cost, the unfriendly impact of the treatment, and patient inclination.

\section{Flutter device in respiratory disorders}

In spite of the fact that the utilization of airway clearance procedures by Pedro et al. [25] is viewed as an imperative part in the treatment of a few obstructive pulmonary diseases, there is no logical proof supporting the utilization of flutter valve in the administration of patients with bronchiectasis. Eight patients were assessed in a randomized, blinded, traverse trial. Impedance at $5 \mathrm{~Hz}$ (R5), resistance as a component of oscillation frequency $(\mathrm{dR} / \mathrm{dF})$, reactance at $5 \mathrm{~Hz}$ (X5), resounding recurrence $\left(\mathrm{f}_{0}\right)$ and necessary of reactance between $5 \mathrm{~Hz}$ and resonant frequency (AX) were recorded. Shudder Valve TM expands sputum evacuation amid treatment and decreases aggregate and fringe airway resistance in hypersecretive patients with bronchiectasis. Drive oscillometry is easy to use the device to assess the impacts of airway clearance systems on respiratory mechanics.

The present devices of physiotherapy for patients with respiratory maladies, were exhibited as an option therapy strategy or a supplemental therapy and they can inspire patients to concern therapy independent from anyone else [26]. These devices appear to build patients' consistency to day by day treatment, since they exhibited numerous benefits, as an autonomous application, full control of therapy and simple utilize. These devices are the positive expiratory pressure, the high-frequency chest wall oscillation, the oral high-frequency oscillation, and the intrapulmonary percussive ventilation, the incentive spirometry the flutter and the acapella and the cornet.

Randomized hybrid trial with disguised portion and aim totreat investigation by Daniela et al. [27]. Here measured the 30 mechanically ventilated patients with aspiratory contamination in a grown-up emergency unit. The exploratory intercession was $30 \mathrm{~min}$ of ventilator-initiated hyperinflation utilizing pressure support ventilation in side-lying; the control mediation was $30 \mathrm{~min}$ of side-lying. Members got the two intercessions around the same time, with a five-hour washout period between them. Result measures: Secretion freedom was measured as sputum volume recovered amid the intercession. Respiratory mechanics were measured as static consistence and aggregate resistance of the respiratory framework prior and then afterward the intercession.

This was a forthcoming, randomized, twofold visually impaired examination that assessed 25 healthy elderly men [28]. The cardiovascular autonomic regulation was evaluated utilizing Heart Rate Variability (HRV) records. The greater part of the volunteers experienced Maximal Inspiratory Pressure 
(MIP) estimations as indicated by institutionalized pulmonary capacity estimations. Three haphazardly connected inspiratory resistive stacking (30, 60 and $80 \%$ of MIP) powers were then connected utilizing an inspiratory resistance device (POWER breathe, Southern, UK), amid which the volunteers were approached to breathe in for $2 \mathrm{~s}$ and breathe out for $3 \mathrm{~s}$ and finish 12 breaths for each moment. Every exertion level was performed for $4 \mathrm{~min}$, and HR and the separation between 2 ensuing $R$ rushes of electrocardiogram ( $R-R$ interims) were gathered very still and at every power for promoting HRV investigation.

Strategies for recuperated cardiac and respiratory parameters utilizing accelerometer and spinner sensors on the wrist are analysed by Javier et al. [29]. Specifically, this indicated it is conceivable to accomplish a mean outright mistake of 1.27 thumps for each moment (STD: 3.37) for heart rate and 0.38 breaths for every moment (STD: 1.19) for breathing rate when contrasting execution and FDA-cleared sensors. Besides, demonstrate equivalent execution with a cutting-edge wristworn heart rate screen, and when checking heart rate of three people amid two back to back evenings of in-situ rest estimations.

\section{Biofeedback Flutter Device in Respiratory Disorders System}

Recently, devices of respiratory physiotherapy have developed which present contrasting options to standard CPT which are less time-consuming and offer more noteworthy autonomy to the patient with a constant lung ailment. As indicated by existing literature, devices of respiratory physiotherapy are presented as option treatment techniques 5-8 with a specific end goal to encourage and enhance the preparation of mucus from the airway, through which better lung ventilation and enhanced pulmonary capacity can be accomplished. The present gadgets appeared in Figure 1 of a respiratory framework having a few devices; it's depicted in beneath the area.

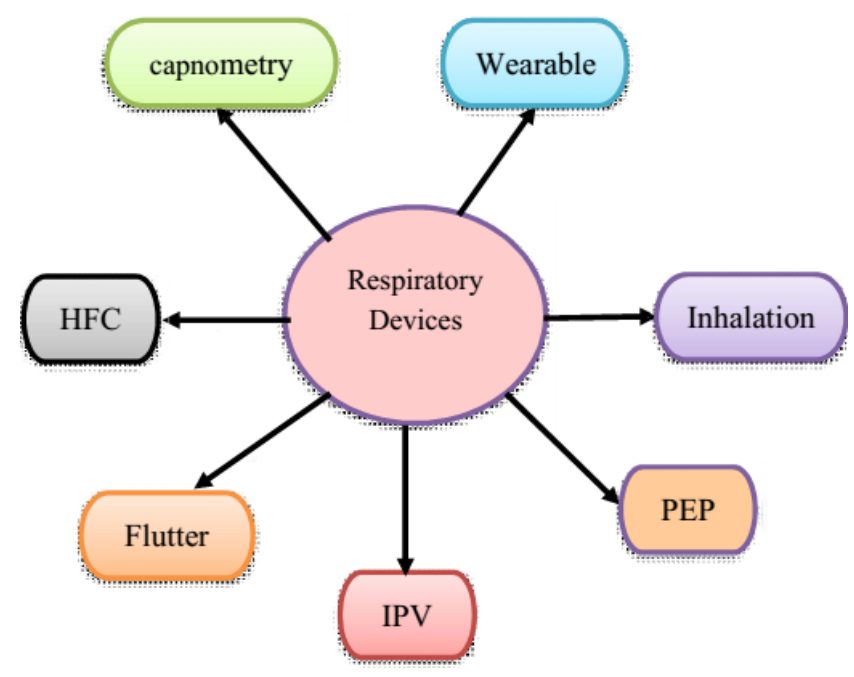

Figure 1. Types of devices in respiratory systems.
Capnometry device: Capnometry is the estimation of carbon dioxide meeting point amid the respiratory cycle. It utilizes infrared innovation to investigate carbon dioxide in exhaled gas. There are numerous choices to encourage sampling of carbon dioxide. The most precise readings are those acquired by testing gasses in the endotracheal container of an intubated patient. Despite that, accompanied by direct and deep sedation the patient is not intubated, so different devices have been produced for gas sampling.

Wearable device: A wearable biofeedback device was amassed utilizing the previously mentioned Body Sensor Network (BSN) modules and related sensors we have created. The device could be utilized as a waistband or a chest band. Within the buckle of the wearable device, there was one BSN node board, diverse sensor interface sheets, and the battery board with a Li-poly rechargeable battery. In the present plan, the heart rate monitoring belt and the breath monitoring belt were made independently, with the goal that it is conceivable to wear one biofeedback device at the thoracic level and another on the abdominal level.

Inhalation device: The right inhaler strategy relies upon the device. Poor inhaler system is tremendously normal and is related to awful results in asthma and COPD, including poor asthma indication control and abuse of relievers and preventers.

High-Frequency chest wall oscillation (HFCWO) device: HFCWO is achieved with a mechanical device (Therapy bronchial drainage system, Hayek Oscillator). Amid HFCWO, positive pressure air pulses are allied to the chest wall, for instance by methods for an inflatable vest and an air pulse generator. The generator generates pressures of around $50 \mathrm{~cm}$ $\mathrm{H}_{2} \mathrm{O}$ at a frequency of approximately $525 \mathrm{~Hz}$ conveyed by means of a pneumatic vest which encompasses the thorax.

Positive Expiratory Pressure (PEP) device: Positive Expiratory Pressure (PEP) treatment was produced in the 1970s and has been presented in the United States as another option to traditional physiotherapy. PEP therapy is the dynamic exhalation of a variable flow resistor achieving pressures of $\sim 10-20 \mathrm{cmH}_{2} \mathrm{O}$. PEP therapy upgrades bronchial hygiene therapy by enhancing airway patency and airflow through airways that are halfway discouraged by stenting the airways as well as expanding intrathoracic pressure distal to held secretions, which: Reduces air- trapping in vulnerable patients; uphold expanded activation and clearance of secretions from the airways. All the devices appear in Figure 2.

Flutter device: The Flutter device was created in Switzerland and joins positive expiratory pressure treatment with highfrequency motions inside the airway 29. It is a prohibited vibration framework which produces positive expiratory pressure and cyclic oscillation of the airways amid expiration. The Flutter device is a convenient device intended to help clear mucus from the lungs.

Application: Flutter device must be utilized as a part of the sitting or supine lying position 42 . The patient is initiated to breathe in profoundly and hold his breath for 2 to $3 \mathrm{~s}$. 
Expiration ought to be slow through the flutter valve; causing oscillations of the steel ball inside the cone of the flutter patients apply rehashed exhalations through the flutter valve. Routinely, three arrangements of 15 exhalations are performed more than 12-20 min. After every arrangement of exhalations, patients were told to "huff" and cough, in this way helping expectoration.

Intrapulmonary percussive ventilation (IPV) device: Intrapulmonary Percussive Ventilation (IPV) is a type of chest physical therapy regulated to the airways by a pneumatic device. Utilizing a breathing circuit termed as Phasitron, smaller than normal blasts of gas is conveyed into the lungs at rates between 100-300 breaths for every moment (bpm). A high yield nebulizer gives a thick aerosol to convey prescriptions and hydrate secretions.

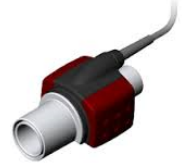

(a) capnometry

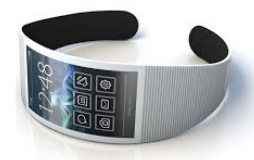

(b) Wearable

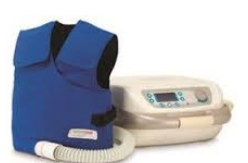

(c) $\mathrm{HFCWO}$

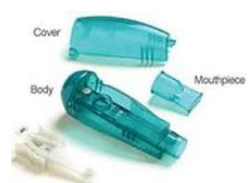

(d) PEP

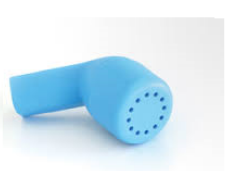

(e) Flutter
Figure 2. Different devices models.

\section{Result and Analysis of Different Articles}

This segment discussed the performance investigation in an assortment of study literature documents to inspect the distinctive devices for respiratory disorder in medical line.
Sequentially, to evaluate the presentation result, primarily the whole documents are grouped gotten from the employed optimization. The successive report utilized hyperinflation methods resounds with physiotherapy literature demonstrating great evidence for improved sputum clearance and lung consistence, and diminished airways resistance. The open investigation inconveniences give up from the expansive function space, which requires framework idea to be versatile.

A few physiotherapy strategies are utilized to encourage a sufficient bronchial drainage in these patients, mainly, dependent upon the patient's consistence and staff mastery. The utilization of devices to expand the clearance of bronchial secretions is generally not considered in the early period of treatment, as these strategies require significant cooperation from the patient. The viability of these devices has not been incorporated into the present audit. In existing examination papers with respect to devices of respiratory physiotherapy distributed too many research papers. The effectiveness of Customary Chest Physiotherapy (CPT) has been affirmed by many examinations. CPT is viewed as the base of respiratory physiotherapy and is portrayed as 'best quality level' of physiotherapy.

Table 1 and Figure 3 demonstrate that the percentages of respiratory devices in distributed papers. In this examination, maximum papers are actualized in flutter devices in $40 \%$ and least count of devices as the HFCWO and capnometry devices. According to published researchers, most of the devices are often used flutter device as 25 studies with percentage $36 \%$, capnometry and Acapella devices are used in 1 study and 1\%, PEP device with 17 studies percentage $25 \%$ and HFCWO device in 10 studies rate as $15 \%$. An inhalation device as 3 papers with $4 \%$ lastly the IPV device 8 considers rate $25 \%$ (Table 2).

Table 1. Devices with measurements.

\begin{tabular}{|c|c|c|c|c|}
\hline Ref number & Device & \multicolumn{3}{|c|}{ Measured parameters } \\
\hline [2] & Wearable device & \multicolumn{3}{|c|}{$\begin{array}{l}\text { Respiration rate, standard deviation of normal to normal (heart rate) SDNN and (LF/HF) Low Frequency and High } \\
\text { Frequency }\end{array}$} \\
\hline [16] & Capnometry & \multicolumn{3}{|c|}{ Length of postoperative hospital stay (LBHS) and risk factors } \\
\hline [22] & Handihaler inhalation device & \multicolumn{3}{|c|}{ Nasal clearance time } \\
\hline [23] & BreatheMAX & \multicolumn{3}{|c|}{ Respiratory rate and changes in secretion clearance } \\
\hline [30] & TPEP device & \multicolumn{3}{|c|}{ Physiological parameters } \\
\hline [24] & In-excitation device & \multicolumn{3}{|c|}{ Pulmonary compliance and $\mathrm{PaO}_{2}$ and a reduction of $\mathrm{MV}$ duration. } \\
\hline [25] & Flutter device & \multicolumn{3}{|c|}{ Standard error of the mean, Relative variance, and expectorated sputum volume } \\
\hline [27] & Positive pressure devices & \multicolumn{3}{|c|}{$\begin{array}{l}\text { Physiological parameters (Mean arterial pressure, mean airway pressures, heart rate, minute ventilation, tidal } \\
\text { volume and respiratory rate.) }\end{array}$} \\
\hline \multicolumn{2}{|c|}{ Table 2. Devices vs. papers. } & & Wearable & 4 \\
\hline Devices & \multicolumn{2}{|c|}{ Count of papers } & Inhalation device & 3 \\
\hline Capnometry & 1 & & HFCWO & 10 \\
\hline
\end{tabular}




\begin{tabular}{ll}
\hline PEP & 17 \\
\hline Flutter & 25 \\
\hline IPV & 8 \\
\hline Acapella & 1 \\
\hline
\end{tabular}

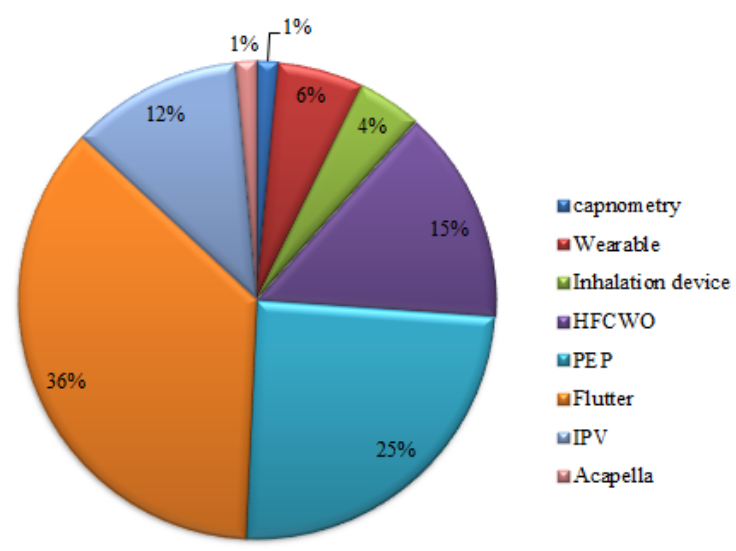

Figure 3. Percentage of deceives sin published articles.

Table 3. Parameters with reference papers.

\begin{tabular}{lllll}
\hline Technique and Ref & $\begin{array}{l}\text { Heart rate } \\
\text { (bpm) }\end{array}$ & $\begin{array}{l}\text { Respiration } \\
\text { Rate (bpm) }\end{array}$ & LF/HF & SDNN (ms) \\
\hline Coherence biofeedback [1] & 90 & - & - & - \\
\hline Wearable device [2] & 75 & 24 & 2.9 & 88 \\
\hline Biofeedback effect [14] & 60 & 24.55 & - & - \\
\hline Biofeedback protocols [15] & 26 & - & - & - \\
\hline Breathing techniques [21] & 58 & 92.3 & 3.2 & \\
\hline $\begin{array}{l}\text { Double blinded crossover } \\
\text { [23] }\end{array}$ & 21 & - & - \\
\hline $\begin{array}{l}\text { BioWatch [25] } \\
\text { Resistive loading protocol }\end{array}$ & 79 & 1.23 & 1.8 & 34 \\
\hline 26] & & & & \\
\hline
\end{tabular}

Table 3 talked about the parameters investigated in various devices and systems required in respiratory disorder. In a few examinations done on postoperative physiotherapy mediation, the part of preoperative physiotherapy in patients experiencing a noteworthy operation, including thoracic surgery, couldn't be totally caught on. As per the parameters of distributed investigations support that more research is expected to characterize the long haul results of the devices of respiratory physiotherapy, to assess patients' consistence to treatment and to underline their impact to personal satisfaction. It is hard to figure out which device of respiratory physiotherapy is better since there are no long haul trials that look at the devices between them.

\section{Heart rate (Bpm)}

Heart rate is the speed of the heartbeat calculated by the quantity of contractions of the heart per minute (bpm). The heart rate can differ as per the body's physical needs, including the need to retain oxygen and discharge carbon dioxide. It is typically equivalent or near the pulse measured at any fringe point. Exercises that can incite change incorporate physical exercise, rest, uneasiness, stress, disease, and ingestion of medications.

\section{Respiration rate (Bpm)}

The respiratory rate in people is measured when a man is at relaxed and includes tallying the quantity of breaths for one moment by checking how often the chest rises. An optical breath rate sensor can be utilized for monitoring patients amid a magnetic resonance imaging scan [1]. Respiration rates may increment with fever, ailment, or other medicinal conditions.

\section{LF/HF}

It has been noticed that HRV (and in this manner LF/HF) is influenced by respiratory parameters and mechanical occasions free of changes in cardiac autonomic nerve activity. The commitment of mechanical components (because of extending of the atria that outcomes from the two changes in the cardiac filling and the changing thoracic pressure that happen amid breath) to changes in HRV.

\section{$\operatorname{SDNN}(M s)$}

Standard Deviation of the Normal-To-Normal intervals (SDNN) reproduces patterns and irregularities in the normal heart rate. SDNN does not allow rough examination of autonomic tone since it fuses all parts of the HRV to a comparative degree.

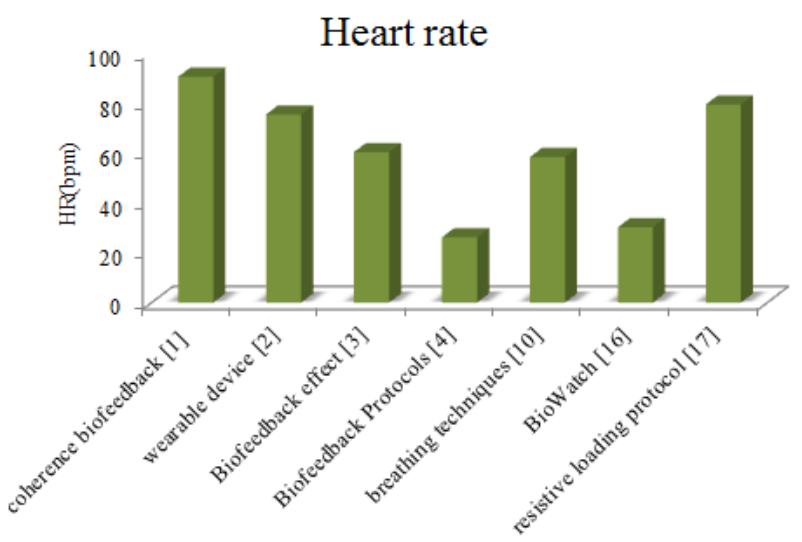

Figure 4. Heart rate analysis.

The advance of the parameter in time area demonstrates the impact of instinctive nature of breathing pattern on heart rate irregularity. Despite the fact that the mean of the length of RR interval doesn't appear to change fundamentally, a number of short HRV sections amid profound breathing test reduced comparing with normal breathing and not common breathing patterns. Figure 4 demonstrates the HR analysis in the various papers examination process. Intelligence biofeedback framework [1] the rate of $99.23 \mathrm{bpm}$ and the base rate as biofeedback convention [15] process. Now the node deviation 
from 20 to 100 , after that the introduction records are $[1,2,14,15,21,26]$. The HRV investigation, that was given, be an examination in a frequency area. Since HRV of healthy subjects acquires to be practically periodical, frequency area techniques are normally more utilized for the correct portrayal of its bend. In order to enhance practices alluringly and productivity, the framework has been created as an actual enjoyment contribution regular ceaseless intelligent 3D movement was identified with the cardiac intelligibility level. In [14] used the biofeedback impact the heart rate as 60 to 70.1 , likewise in all papers. At that point the bio looks as the one of the vacillate device [25] it's resolved the better heart rate.

\section{Respiration Rate}

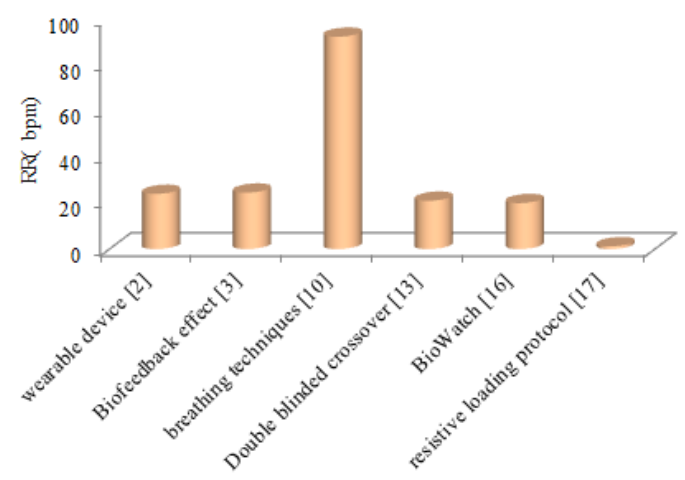

Figure 5. Respiration rate analysis.

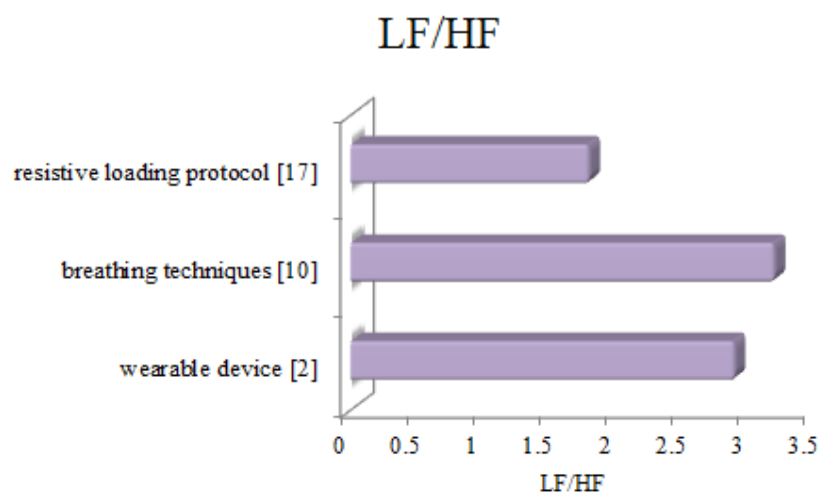

Figure 6. LF/HF analysis.

Respiration signal acquired from the magnetic displacement sensors and the concurrently recorded ECG from a subject breathing at $8 \mathrm{bpm}$. The amplitude of the $\mathrm{R}$ wave and the RR interval are unmistakably regulated at a rate comparing to the respiratory signal. The respiratory rates gotten from the central part inferred breath were precise and the contrasts amongst these and the deliberate respiratory. As the algorithm necessitates three maxima it will be three breaths behind at the on-going respiratory rate. This technique has been picked as opposed to taking more estimations and ascertaining its weighted normal after some time since this would give a factual respiratory rate, which was inquired about in [17], rather than a constant one. Figure 5 demonstrates the RR of a few papers like $[2,14,15,23,26]$. The minimum $R R$ in resistive loading protocol [26] and greatest value achieves in breathing method [21]. Therefore, despite the fact that our sample did not present respiratory muscle shortcoming contrasted with the reference esteems, we trust that future examinations including this populace with inspiratory shortcoming will be advantageous for more prominent comprehension of the autonomic HR reactions.

LF and HF control is assessed as the area under a range of HRV in the frequency ranges appeared in Figure 6. The LF/HF control is the scientific proportion of LF energy to HF control. The proportion is restored each $5 \mathrm{~s}$ and given to students in sound and chart shape. The sound is an unadulterated tone which changes its frequency each $5 \mathrm{~s}$ and goes on for $500 \mathrm{~ms}$. the frequency of sound is chosen by the LF/HF proportion. At the point when the proportion expands the frequency as needs be increments, on the other hand, if apportion diminish the frequency diminishes as well. The diagram is blue bar outlines which change the stature as per the LF/HF proportion. The figure demonstrates the LF/RF proportion of responsorial framework the most extreme proportion as [21] in breathing strategies.

\section{Conclusion}

Biofeedback and airway clearance has been utilized for a long time to help patients and clinicians among recovery. This paper has checked on the biofeedback distinctive devices with application in flutter devices that are presently being utilized as a part of physical rehabilitation and classified the diverse sorts of biofeedback into two primary classes; physiological biofeedback, biomechanical biofeedback. The number of published reports is restricted; however, more research is expected to characterize the adequacy of the devices of respiratory physiotherapy and their places with the current procedures are accessible. Complete validation of two physiological estimations (HR, BR) in a controlled research center setting gave preparatory approval of heart rate estimation from the wrist-worn movement detecting in an actual rest setting and showed enhanced accuracy when consolidating wearable light-based and movement based sensors. Additionally, the patient must be educated that these devices must not supplant the programmed of respiratory physiotherapy, since they have a supplemental part to respiratory physiotherapy, adding to better outcomes in pulmonary function. From the review, investigation demonstrates that the flutter devices are the best of respiratory disorders in biofeedback examination. Along these lines, we need to modernize the imaginative technologies every day. Furthermore, we think about that as a prevalent amount of works will be upgraded in prospect through using our investigation work. Hence, from this review analysis, we determined various biofeedback systems complied with devices, whereas the devices used for respiratory disorders which help in mucus clearance does not add any biofeedback systems. Further, we conclude the review by adding that biofeedback respiratory devices could enhance the 
performance of patients and good effects over health can be obtained and relieve stress.

\section{References}

1. Ibarissene IMF, Logier R. A smartphone-based cardiac coherence biofeedback system. Engineering in Medicine and Biology Society (EMBC), 2014 36th Annual International Conference of the IEEE 2014; 4791-4794.

2. Liu GZ, Bang YH, Zhan YM, Yan WG, Lei W. A wearable respiratory biofeedback system based on body sensor networks. Engineering in Medicine and Biology Society (EMBC), 2010 Annual International Conference of the IEEE 2010; 2497-2500.

3. Button BM, Christine W, Ruth D, Narelle SC, Anna M, Esta T, Jennifer B. Physiotherapy for cystic fibrosis in Australia and New Zealand: A clinical practice guideline. Respirology 2016; 21: 656-667.

4. Shi Y, Shuai R, Jinglong N, Maolin C, Weiqing X. An intelligent secretion clearance system based on pneumatics. Fluid Power and Mechatronics (FPM), 2015 International Conference IEEE 2015; 355-359.

5. Uhlmann C, Froscher W. Biofeedback treatment in patients with refractory epilepsy: changes in depression and control orientation. Seizure 2001; 10: 34-38.

6. Naraparaju SKV, PremV, Vishak A. A comparison of the Acapella and a threshold inspiratory muscle trainer for sputum clearance in bronchiectasis-a pilot study. Physiother Theor Pract 2010; 26: 353-357.

7. Moravec CS, Michael GM. Biofeedback in the treatment of heart disease. Cleveland Clin J Med 2011; 78: 20-23.

8. Bernardi L, Porta C, Spicuzza L, Bellwon J, Spadacini G, Frey AW, Yeung LY, Sanderson JE, Pedretti R, Tramarin R. Slow breathing increases arterial baroreflex sensitivity in patients with chronic heart failure. Circulation 2002; 105: 143-145.

9. Chatburn RL. High-frequency assisted airway clearance. Resp Care 2007; 52: 1224-1237.

10. Kuper CF, Grollers-Mulderij M, Maarschalkerweerd T, Meulendijks NM, Reus A, van Acker F, Zondervan-van den Beuken EK, Wouters ME, Bijlsma S, Kooter IM. Toxicity assessment of aggregated/agglomerated cerium oxide nanoparticles in an in vitro 3D airway model: the influence of mucociliary clearance. Toxicol In Vitro 2015; 29: 389-397.

11. Yi WJ, Park KS, Lee CH, Rhee CSr. Simultaneous measurement of ciliary beat frequency and wave disorder on respiratory epithelial cells. Engineering in Medicine and Biology Society, 2003. Proceedings of the 25th Annual International Conference of the IEEE 2003; 2: 1047-1049.

12. Bennett WD, Aline KH, Duckworth KLZ, Jihong W, Ashley H, Melissa Y, Bruce KR. Effect of a single $1200 \mathrm{Mg}$ dose of Mucinex ${ }^{\circledR}$ on mucociliary and cough clearance during an acute respiratory tract infection. Resp Med 2015; 109: 1476-1483.
13. Denehy L, Susan B. Physiotherapy in the intensive care unit. Phys Ther Rev 2006; 1: 49-56.

14. Bhandari R, Avinash P, Eva S, Beena A, Ricardo GO. Music-based respiratory biofeedback in visuallydemanding tasks. NIME 2015; 78-82.

15. Munafo MDP, Casarotti M. Biofeedback protocols for stress management in high-performance work environments. Measur Behav 2014; 1-5.

16. Grishin OV, Gultyaeva VV, Zinchenko MI, Yu Uryumtsev D, Zhilina IG, Grishin VG. Feasibility of a capnometry device for respiratory biofeedback in patients undergoing coronary artery bypass graft surgery. Biomedical Engineering and Computational Technologies (SIBIRCON), 2015 International Conference IEEE 2015; 22-26.

17. Gosselink R. Physical therapy in adults with respiratory disorders: where are we? Braz J Phys Ther 2006; 10: 361-372.

18. Sudhir GK, Smitha N, Narasimha Swamy KN, Vinay HR. Biofeedback as an assessment tool in measuring effectiveness of alternate nostril breathing (NadiShodhana Pranayama) amongst medical students. J Dent Med Sci 2015; 14: 1-3.

19. Giggins OM, Persson UM, Caulfield B. Biofeedback in rehabilitation. J Neuroeng Rehab 2013; 10: 1-12.

20. Munafo M, Elisabetta P, Daniela P. Improving managers psychophysical well-being: effectiveness of respiratory sinus arrhythmia biofeedback. Appl Psychophysiol Biofeedback 2016; 41: 129-139.

21.Zhu Q, Fei Z, Ying-ying X. Respiratory training biofeedback system. Electronics, Communications and Control (ICECC), 2011 International Conference IEEE 2011; 3915-3918.

22. Tagaya E, Osamitsu Y, Akitoshi S, Ken A, Kiyoshi T, Mitsuko K, Jun T. Effect of tiotropium on mucus hypersecretion and airway clearance in patients with COPD. Pulm Pharmacol Ther 2016; 39: 81-84.

23. Jones CU, Sujittra K, Seksan C, Wilaiwan K. Breathing exercise using a new breathing device increases airway secretion clearance in mechanically ventilated patients. Heart Lung J Acute Crit Care 2013; 42: 177-182.

24. Makhabah D, Nicolino A. Airway clearance in the intensive care unit. EMJ Respir 2013; 1: 135-139.

25. Figueiredo Pedro HS, Walter AZ, Fernando SG. Flutter valve improves respiratory mechanics and sputum production in patients with bronchiectasis. Physiother Res Int 2012; 17: 12-20.

26. Hristara-Papadopoulou A, Tsanakas J, Diomou G, Papadopoulou O. Current devices of respiratory physiotherapy, Hippokratia 2008; 12: 212-222.

27. Lemes DA, Walter A, Fernando SG. Hyperinflation using pressure support ventilation improves secretion clearance and respiratory mechanics in ventilated patients with pulmonary infection: a randomised crossover trial. Aus J Physiother 2009; 55: 249-254. 
28. Archiza B, Rodrigo PS, Renata GM, Guilherme AFF, Aparecida MC, Audrey BS. Acute effects of different inspiratory resistive loading on heart rate variability in healthy elderly patients. Braz J Phys Ther 2013; 17: 401-408.

29. Hernandez J, Daniel M, Rosalind WP. Biowatch: estimation of heart and breathing rates from wrist motions. Proceedings of the 9th International Conference on Pervasive Computing Technologies for Healthcare 2015; 169-176.

30. Dabrosca F, Barbara G, Gloria S, Agnese B, Lorenzo A, Luis VFO, Paola B, Bruno B. Comparing airways clearance techniques in chronic obstructive pulmonary disease and bronchiectasis: positive expiratory pressure or temporary positive expiratory pressure? A retrospective study. Braz $\mathrm{J}$ Phys Ther 2017; 21: 15-23.

\section{*Correspondence to}

Rekha k

Saveetha College of Physiotherapy

Tamil Nadu

India 